Meta

Journal des traducteurs

Translators' Journal

\title{
Index of Words and Topics in Volume 21
}

Volume 21, numéro 4, décembre 1976

URI : https://id.erudit.org/iderudit/003188ar

DOI : https://doi.org/10.7202/003188ar

Aller au sommaire du numéro

\section{Éditeur(s)}

Les Presses de l'Université de Montréal

ISSN

0026-0452 (imprimé)

1492-1421 (numérique)

Découvrir la revue

Citer ce document

(1976). Index of Words and Topics in Volume 21. Meta, 21(4), 305-307.

https://doi.org/10.7202/003188ar d'utilisation que vous pouvez consulter en ligne.

https://apropos.erudit.org/fr/usagers/politique-dutilisation/ 


\section{INDEX OF WORDS AND TOPICS IN VOLUME 21}

Abstracting, 2, p. 149.

Abstract journal, 2, p. 147

Action-oriented sign system, 4, p 246.

Adjective + computer, 4 , p. 263 .

Advance payment, 1, p. 112 .

Affect (to), 4, p. 274.

Agricultural labourer, 4, p. 278.

Agricultural science, 4, p, 281.

Agriculture, 4, p. 278

Agronomy, 4, p. 281.

Aim of translation, 4, p. 235.

Alerting service, 2 , p. 147.

All weather track, 3, p. 216.

Animal feed, 4, p. 279.

Anisomorphism, 4, p. 236.

Assembly, 4, p. 237.

Assessing translation quality, 4, p. 294.

Aviation spirit, 2, p. 163.

Baal Seminar on translation, 4, p. 294.

Batch processing, 2, p. 147

Bearing an index, 4 , p. 275.

Bibliography (linguistics), 4, p. 249.

Bibliography (solid-state), 4 , p. 273.

Big, 4, p. 242.

Billion (U.S. and G.B.), 1, p. 92.

Binghampton, 2, p. 179.

Binominal expressions from English into French, 4, p. 294.

Black rot, 4, p. 283.

Blend, 4, p. 279.

Boom, 4, p. 284.

Brand name, 3, p. 197.

Breed, 4, p. 280.

Calculator, 4, p. 261.

Cardioid, 4, p. 284.

Case marker, 4, p. 242.

Check-off, 3, p. 213

Chip, 4, p. 262.

Citation, 2, p. 147.

Citation index, 2, p. 147.

Clap, 4, p. 284.

Clearing house, 2, p. 147

Clear period, 2, p. 130.

Clouding over, 2, p. 130

Club wheat, 4 , p. 280.

"Coke ", 3, p. 200.

Common wheat, 4, p. 281.

Comparing translations, 4, p. 294.

Compliments (with), 2, p. 161.

Componential analysis, 4, p. 242, 294.

Compound semiconductor material, 4, p. 266.

Computer, 4 , p. 261.

Computerized information retrieval, 2, p. 147.

Computer specialist, 4, p. 259.

Controlled price, 4 , p. 281.

Controlled vocabulary, 2, p. 148.

Conversational, 2, p, 148

Copyright, 2, p. 141

Corn earworm, 4, p. 283.

Cow testing, 4, p. 278 .

Cultural filter, 4, p. 295
Cultural overlap, 4, p. 236.

Cultural voids, 4 , p. 238.

Current awareness, 2, p. 147, 148.

Cut, 4 , p. 284

Data bank, 2, p. 148.

Data base, 2, p. 148.

Data processing expert, 4 , p. 259

Demetaphorized compound, 4, p. 244.

Denominational meaning, 4, p. 237.

Depository, 2, p. 148.

Display, 2, p. 148

Distributor's brand, 3, p. 202.

Dope-sheet, 4, p. 284.

Downturn, 4, p. 286.

Durum wheat, 4, p. 281.

Dynamic level of language, 4, p. 237.

Environmental voids, 4, p. 238.

Ergative constructions, 4, p. 241.

Farm hand, 4, p. 280

Fattening feed, 4 , p. 282.

Feature, 4, p. 284.

Feed, 4, p. 279

Feedback, 2, p. 149

Feed grain, 4, p. 282.

Fertilizer blend, 4, p. 279.

Field. 2, p. 149.

Field crop, 4, p. 282.

Figurative usage, 4, p. 242.

Filister head, 1, p. 92.

Financial year, 1, p. 112

Fixed flexible working hours, 3, p. 207.

Fixed working hours, 3, p. 206.

Flexible working hours, 3, p. 206.

Flint wheat, 4, p. 281.

Fodder, 4, p. 279.

Food, 4, p. 279.

Forage seed, 4, p. 282.

Formatting, 2, p. 149.

French group, 1, p. 64.

French services, 1, p. 64.

Fringe benefits, 4, p. 285.

Frozen price, 4 , p. 281.

Full shot, 4, p. 284.

Gender markers, 4 , p. 240

Geneticist, 4, p. 280.

Genetics, 4, p. 280

Goldenrod, 3, p. 214.

Granulated feed, 4, p. 279

Guided ground transport, 2, p. 165.

Hard wheat, 4, p. 281.

Hay tedder, 4, p. 282.

Heavy fog, 2, p. 132.

Heavy roin, 2 , p. 132

Historical voids, 4, p. 239.

Idiomatic anisomorphisme, 4, p. 244.

Indexing, 2, p. 149.

Indexing journal, 2, p. 149

Individual working time, 3, p. 207.

Information retrieval, 2, p. 149.

Integrated circuit, 4 , p. 268.

Interactive, 2, p. 149. 
Interest profile, 2, p. 149.

International Poetry Forum, 3, p. 229.

Isolated cloudy period, 2, p. 132.

Know-how, 4, p. 285

Laborer, 4, p. 280

Labour force, 4, p. 280.

Lacking word, 4 , p. 288.

Language fatigue, 4, p. 288

Legumes, 4, p. 279.

Leguminous crops, 4, p. 279.

Lexical anisomorphism, 4, p. 237.

Lexical voids, 4, p. 239.

Line, 4 , p. 280

Line breading, 4, p. 280.

Linguistics and translation, 4, p. 294.

Linguistic social competence, 4, p. 247.

Literary translation, 2 , p. 165.

Literature, 2, p. 150.

Literature search, 2, p. 150

Loan translation, 4, p. 244.

Logical operator, 2, p. 150.

Log off, 2, p. 150.

Log on, 2, p. 150

Macaroni wheat, 4, p. 281.

Machine-readable, 2, p. 150.

Markers, 4, p. 240.

Match, 2, p. 150.

Metaphor, 4, p. 243.

Microcomputer, 4, p. 262.

Middleman's brand, 3, p. 202.

Milk replacer, 4, p. 282.

Mistranslation of a feature of style, 4, p. 295.

Mixed feed, 4, p. 279.

Mixed fertilizer, 4, p. 279

Mixed fodder, 4, p. 279.

Model for assessing translation quality, 4, p. 294

Mortgaged, 4, p. 275.

Motor vehicle, 2, p. 164

Mumbo jumbo, 1, p. 84 .

Mushrooms, 3, p. 214.

Naming and address, 4, p. 236.

Nasals, 2, p. 168

Nation, 4, p. 237

Nationality, 4, p. 237.

Near dawn, 2, p. 131.

Not-equal form, 4, p. 236

Occasional cloudy period, 2, p. 132.

Off-line, 2, p. 150.

Off-type, 4, p. 282

Onomatopoeia, 4, p. 239.

“Parisian French ", 1, p. 58.

Pelleted feed, 4, p. 279.

Pellets, 4, p. 279.

People, 4, p. 237.

Personal income tax, 2, p. 163.

Phonic values, 2 , p. 167.

Phonological voids, 4, p. 239.

Piano, 2, p. 143.

Piano lexicon, 2, p. 145.

Pirate, 2, p. 141

Pirate recording, 2, p. 141.

Pirate station, 2, p. 141.

Plant breeder, 4, p. 280.

Plant breeding, 4, p. 280.

Plant food, 4, p. 279.

Plurality markers, 4, p. 241

Poetry, 2, p. 165

Polysemy, 4, p. 242.
Prestige sound, 4, p. 239.

Price control, 4, p. 281.

Price monitoring, 4, p. 281.

Printout, 2, p. 150.

Privale brand, 3 , p. 197.

Professional voids, 4, p. 238.

Profile design, 2, p. 151.

Pulse crops, 4, p. 279.

Pulses, 4, p. 279.

Quality of translation, 4, p. 295.

Race, 4, p. 237, 280.

Radiacmeter, 4, p. 257.

Reaper, 4, p. 282

Recall, 2, p. 151.

Reference, 2, p. 151.

Reference work, 2, p. 151

Referential meaning, 4, p. 237.

Referential synonyms, 4, p. 238.

Referral, 2, p. 151.

Reformat, 2, p. 152.

Registered trademark, 3, p. 199.

Religious voids, 4 , p. 238 .

Retrieve, 2, p. 152.

Rock maple, 4, p. 279.

Scientific terminology, 4, p. 236.

Script-girl, 4, p. 284

Search against, 2, p. 152.

Search editor, 2, p. 152.

Search expression, 2, p. 152.

Search formulation, 2, p. 152.

Search logic, 2, p. 152 .

Search strategy, 2, p. 152.

Selective dissemination of information, 2, p. 152.

Semantic competence, 4, p. 242.

Semantic voids, 4 , p. 238.

Sememic level, 4, p. 242.

Semiconductor battery, 4, p. 271.

Semiconductor material, 4 , p. 266

Sex markers, 4 , p. 240

Sign off, 2, p. 153 .

Sign on, 2, p. 153.

Single ingredient feed, 4, p. 279.

Situational identities, 1, p. 246

Sleeping policemen, 4, p. 257.

Social voids, 4 , p. 238 .

Soft wheat. 4, p. 281

Solids non fat, 4 , p. 282

Solid state, 4, p. 264

Solid-state battery, 4, p. 271

Solid-state circuit, 4, p. 267.

Solid-state component, 4, p. 266.

Solid-state computer, 4, p. 268

Solid-state device, 4, p. 266.

Solid-state integrated circuit, 4, p. 268.

Solid-state laser, 4, p. 268.

Solid-state maser, 4 , p. 268

Solid-state materials, 4, p. 265

Solid-state physics, 4, p. 265.

Solid-state rectifier, 4, p. 269.

Solid-state relay, 4, p. 267.

Solid-state static alternator, 4, p. 269.

Solid-state television, 4, p. 271.

Solid state vocabulary, 4 , p. 272

Species, 4, p. 237.

Spirit, 2, p. 163.

Staggered day work, 3, p. 206.

Staggering of hours, 3, p. 206.

Statements of case, 1, p. 112. 
State-of-action marker, 4, p. 241.

Static level of language, 4, p. 237.

Stock feed, 4, p. 279.

Storage, 2, p. 153.

Strain, 4, p. 280.

Stratificational voids, 4 , p. 238

Style, 4, p. 245

Stylistic anisomorphism, 4, p. 245.

Sugar bush, 4, p. 279.

Sugar maple, 4, p. 279.

Sugar plant, 4, p. 280

Supervisor, 3, p. 213.

Supervisory personnel, 3, p. 213.

Supply, 2, p. 163

Synchronization, 4, p. 286.

Syntactic markers, 4, p. 240.

Syntactic voids, 4, p. 239

Tape services, 2, p. 153

Tartan, 3, p. 216.

Terminal, 2, p. 153.

Text typology, 4, p. 295.

Textual profile, 4, p. 294.

Textual structure difference in English and German, 4, p. 294
Theory of human language, 4, p. 235.

Time-sharing, 2, p. 153.

Tomato fruitworm, 4, p. 283

Trademark, 3, p. 197.

Trade name, 3, p. 197.

Training of translators, 4, p. 295.

Transcreator, 2, p. 166.

Transformer, 4, p. 276.

Translation model, 4, p. 235.

Translation theory, 4, p. 235

Tribe, 4, p. 237.

Triticum compactum, 4, p. 280.

Truncation, 2, p. 153.

User, 2, p. 153.

Vegetable market, 4, p. 278.

Void, 4, p. 236.

Washer head, 1, p. 92

Week-end, 4, p. 287.

Weighting, 2, p. 154 .

Word authority list, 2, p. 154.

World views, 4, p. 236.

Zero representation, 4, p. 241. 